International Journal of Distributed and Parallel Systems (IJDPS) Vol.3, No.3, May 2012

\title{
THROUGHPUT IMPROVEMENT IN WIRELESS MESH NETWORKS BY INTEGRATING WITH OPTICAL NETWORK
}

\author{
Chakrapani gadde, K.Chandrasekhar and I.Hemalatha \\ Department of Electronics and Communications, SIR CRR college of engineering, \\ Eluru,India. \\ chakri.gadde@gmail.com, chandu_sekhar491@yahoo.co.in, \\ hemacrr_2006@yahoo.co.in
}

\begin{abstract}
In the last decade wireless mesh networks (WMNs) have emerged as a key technology for next generation wireless networking. Because of their advantages over other wireless networks, WMNs are undergoing rapid progress and inspiring numerous applications. One such application is to provide peer-to-peer communication for all the users who are distributed over some area. Since the users are connected in a wireless multi-hop passion complete ubiquity is provided. But as the number of users accessing the network is increasing there could be a chance of experiencing more interference by each user due to the communication link of every other user. So in a wireless mesh network as the load increases the throughput of network is going to be decreased due to wireless interference by other users. To sustain this problem we are going to integrate the WMN with passive optical network (PON). The resulting hybrid network (Optical-wireless network) could reduce the wireless hops of each user, so that we can reduce the total wireless interference experienced by each user resulting in improved network throughput. This paper aims to study the network throughput gain in Optical-wireless network subject to peer-to-peer communications.
\end{abstract}

\section{KEYWORDS}

Wireless mesh network, passive optical network, Optical-wireless network, peer-to-peer communication, throughput.

\section{INTRODUCTION}

Optical and wireless access networks were originally developed for different communication scenarios. Optical networks aim to provide long distance, high-bandwidth communications [2] while wireless networks aim to provide ubiquitous, flexible communications mainly in community areas [1]. Various kinds of optical and wireless access network architectures have been proposed and deployed as solutions for access networks separately. A WMN typically consists of multiple gateways for the Internet access, a group of wireless mesh routers supporting multi-hop communications, and a group of mesh clients associated with wireless mesh routers as shown in figure 1. Nowadays, Passive Optical Network (PON) [2] has emerged to become the most popular optical access network solution. A PON typically consists of an Optical Line Terminal (OLT) at the central office transmitting optical signals received from the access network to the Internet and vice versa, and a group of Optical network Units (ONUs) receiving downstream optical signals from the OLT and generating upstream optical signals to the OLT as shown in figure 2. For wireless access networks, Wireless Mesh Networks (WMNs) [1] extend the reach of Wireless LANs cost effectively and have been widely deployed at DOI : 10.5121/ijdps.2012.3320 
enterprises and community areas. In view of complementary features of both optical and wireless networks, the integrated optical-wireless (and WOBAN [3]) network has been proposed to provide a high bandwidth, low-cost hybrid access network as shown in Figure 3. The integration equips ONUs with the capability of modulating wirelessly received signals from wireless mesh routers to upstream optical signals transmitted to the OLT and the capability of demodulating optical signals received from the OLT and then wirelessly transmitting the signal to the WMN. In other words, ONUs in Optical-wireless network combines the function of traditional ONUs in PONs and gateways in WMNs together. An alternative implementation of ONUs in Optical-wireless network is to connect traditional ONUs in PONs with gateways in WMNs through wired links.

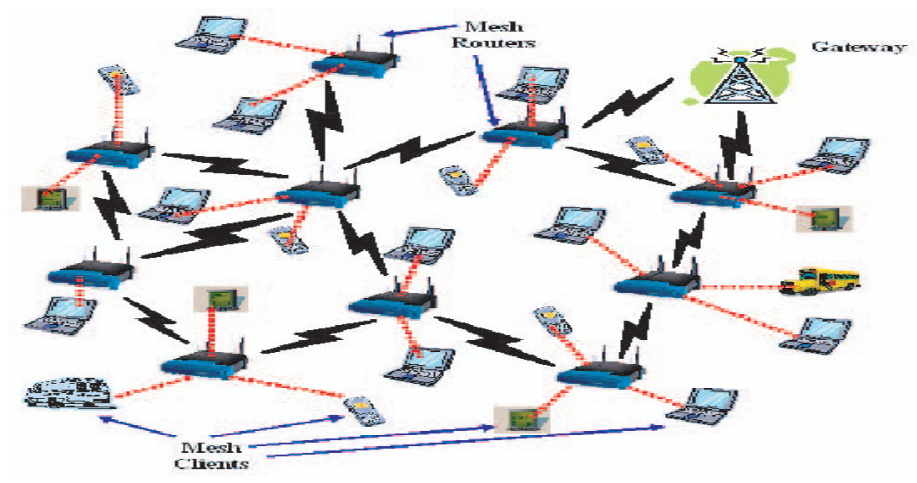

Figure 1. Wireless mesh network

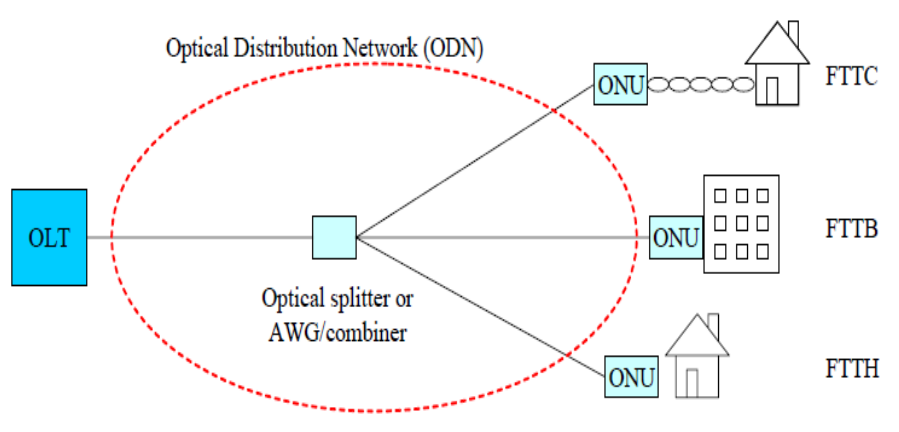

Figure 2. General PON architecture

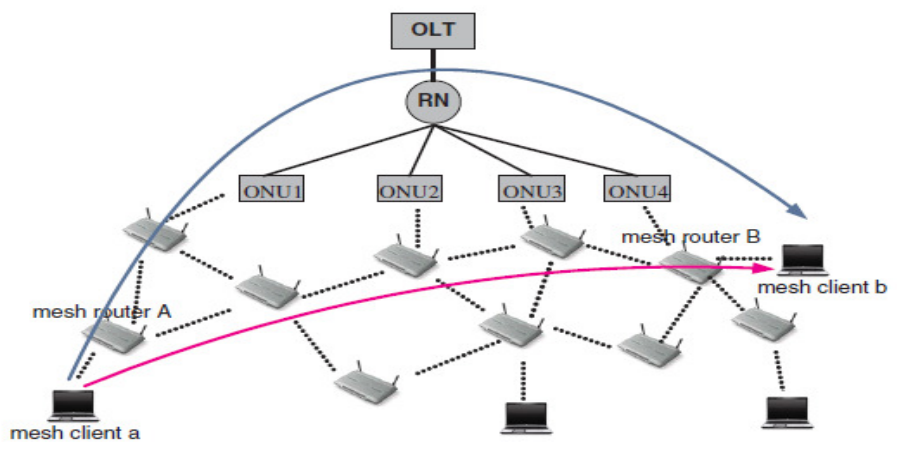

Figure 3. Architecture of optical-wireless network 
International Journal of Distributed and Parallel Systems (IJDPS) Vol.3, No.3, May 2012

Although PON can provide high bandwidth, e.g., 1.25Gbps EPON, the network throughput of the Optical-wireless network is still bottlenecked by the interference in wireless communications. In a single-radio single-channel wireless network, the maximum throughput at each gateway will be much less than the radio's data rate due to intra-path and inter-path interferences. However, the integration of PONs and WMNs in Optical-wireless networks provides an opportunity to reduce the impact of interferences on the network throughput, especially for peer to peer communications from one wireless client to another wireless client. Nowadays, the growth of applications such as multimedia communications, Voice and Video over IP (VoIP) have made peer-to-peer communications increasingly useful in which two wireless clients can do things such as video transmitting, online chatting etc..., if a mesh client would like to communicate with another mesh client, traffic will be routed through the multihop wireless path in WMNs. However, in Optical-wireless networks, such traffic can be routed from source mesh client to its nearest ONU, which then sends the traffic to the OLT, the OLT then broadcasts the traffic back to ONUs, the ONU closest to destination client can then send the traffic to destination client. Such a wireless- optical- wireless communication for peer-topeer traffic in Optical-wireless networks can bring following advantages:

(i) It can potentially reduce the delay for some peer-to-peer communications since the transmission in the PON sub network can be much faster than that in the multi-hop wireless network.

(ii) If some peer-to-peer communications are rerouted through the wireless-optical-wireless manner, such traffic may cause less interference with other communications.

(iii)

We can extend the reach of wireless mesh network.

Such observations motivate the study of this paper. In this paper, we aim to study the network throughput gain in Optical-wireless networks subject to peer-to-peer communications. The rest of the paper is organized as follows. Section 2 introduces the related work. Section 3 explains the system model and assumptions. Section 4 presents the routing algorithm for throughput optimization and section 5 shows simulation results. We conclude the paper in section 6 .

\section{RELATED WORK}

Recently, the hybrid Optical-wireless network has become a very attractive topic. Work in this research area has been focusing on the optimal placement for ONUs, the reconfiguration of PON structures and routing algorithms in the wireless sub network of Optical-wireless network. Mixed Integer Programming (MIP) Model [4] and Simulated Annealing (SA) algorithm [5] have been proposed to minimize the average distance of any wireless mesh router to its neighbourhood ONU. A dynamic reconfiguration algorithm in WDM PONs is proposed in [6] for the better bandwidth utilization. Delay-Aware Routing Algorithm (DARA) [7] and Capacity and Delay Aware Routing Algorithm (CaDAR) [8] have been proposed to address the routing issue in the Optical-wireless network. Moreover, a centralized integrated routing algorithm is proposed in [9] to achieve the load balance at ONUs and to maximize the network throughput. In order to evaluate the network throughput gain in Optical-wireless networks, issues like network capacity, traffic routing and channel assignment in the wireless mesh sub network play important roles. In the following, we would like to introduce the related work in WMNs on those aspects. In the pioneer work, [9] shows that in a wireless network with $n$ identical nodes, the per-node throughput is $\Theta\left(\frac{1}{\sqrt{n l o g n}}\right)$ assuming the random node placement and communication pattern. It becomes $\Theta\left(\frac{1}{\sqrt{ }}\right)$ assuming the optimal node placement and communication pattern.

The work in [10] aims to maximize the network throughput and meanwhile to guarantee the fairness among traffic flows destined to multiple gateways. Both [11] [12] propose LP based routing algorithms and channel assignment algorithms in multi-radio, multi-channel WMNs. In 
particular, [11] solves the joint routing and channel assignment problem which intends to optimize the overall network throughput subject to the fairness constraint. In general, most work on both Optical-wireless networks and WMNs focus on the traffic destined to gateways for the Internet access. As introduced in the previous section, Optical-wireless network provides an attractive alternative routing for peer-to-peer communications, which can utilize the high bandwidth provided by the PON sub network and alleviate interferences in wireless sub network, thus increasing the network throughput. Therefore, our focus is on the network throughput gain brought by the new structural features in Optical-wireless networks compared with traditional WMNs when peer-to-peer communications is the traffic load.

\section{SYSTEM MODEL AND ASSUMPTIONS}

\subsection{Basics}

We model a Optical-wireless network as a directed graph $G=(N, E)$ where $N$ consists of the OLT denoted as node $u_{0}$, a set of ONUs (gateways) denoted as $N_{G}$ and a set of wireless mesh routers denoted as $N_{M}$. We assume that each wireless router is equipped with one radio working on the single channel with channel capacity $c$. All wireless nodes use the same fixed transmission power. The transmission range and the interference range are $R_{t}$ and $R_{i}$ respectively. Typically, $R_{\mathrm{i}}$ is $\beta$ times of $R_{t}$ where $\beta \geq 1$. For any two nodes $\mathrm{u} \in N_{M} \cup N_{G}$ and $v \in$ $N_{M}$, there are wireless links $e_{u v}, e_{v u} \in E$ if and only if $u$ is within $v$ 's transmission range and vice versa. All wireless nodes are assumed to be stationary. In Optical-wireless networks, ONUs (gateways) can communicate with each other by sending traffic to the OLT which then broadcasts traffic back to all ONUs. Since the capacity in the PON sub network is much larger than that in the wireless sub network, we can model that there is a direct communication between any two ONUs with infinite capacity. In other words, for any two nodes $u, v \in N_{G}$, there are links $e_{u v}, e_{v u} \in E$ with infinite link capacity. Since such communication happens in the PON sub network, there is no wireless interference and the interference set of such links is set to be $\Phi$.

Since our aim is to compare the throughputs of wireless mesh network and optical wireless network, for fair comparison we design a wireless network as a direct graph $\mathrm{G}=(\mathrm{N}, \mathrm{E})$ where $\mathrm{N}$ consists of a set of wireless mesh routers only. We assume that each wireless router is equipped with one radio working on the single channel with channel capacity $\mathrm{c}$ as we considered in optical-wireless network. The transmission range and interference range are also same as optical-wireless model. In wireless mesh network communication links exist only among the wireless routers i.e. for any two nodes $u \in N_{M}$ and $v \in N_{M}$, there are wireless links $e_{u v}, e_{v u} \in E$ if and only if $u$ is within v's transmission range and vice versa.

\subsection{Interference model}

In our work, CSMA/CA with RTS/CTS is adopted to handle the MAC layer data transmissions in the wireless sub network of Optical-wireless networks. Therefore, the transmission on wireless link $e_{u v}$ may prohibit data transmissions within $R_{i}$ away from both node $u$ because of RTS and node $v$ because of CTS. For each node $u \in N_{M} \cup N_{G}$, assume that there is an interference disk $D_{u}$ centered at $u$ with radius $R_{i}$. For any wireless transmitting link $e_{u v}$, we denote the union area of $D_{u}$ and $D_{v}$ as $D_{u v}$ and regard any wireless transmitting link $e_{x y}$ with its transmitting node $x$ located in the area $D_{u v}$ as the interference link of $e_{u v}$. In particular, we denote Inter $\left(e_{u v}\right) \subseteq E$ as the set of Wireless links that interfere with $e_{u v}$.

\section{Traffic Routing}

In both wireless mesh and Optical-wireless network, since we assume that each node has only one radio working on one channel, there is no channel assignment issue. In order to derive the 
maximum network throughput, we only need to consider routing and link scheduling issues. Considering the routing issue in traditional WMNs, traffic for peer-to-peer communications will always go through the shortest path between peer clients within the wireless network. On the other hand, in optical-wireless networks, for peer-to-peer communication between two clients, traffic will be first routed from source client to its nearest ONU through the shortest path within the wireless sub network. Then in the PON sub network, traffic will be sent to the OLT which then broadcasts traffic back to all ONUs. The ONU closest to destination client then sends traffic to the destination client through the shortest path within the wireless sub network again. In case if destination client is reached even before the ONU, traffic is directly routed to destination client from source client. In this case routing of the traffic will be same as traditional wireless mesh network.

\section{Evaluation}

In this section, we evaluate the network throughput gain in Optical-wireless networks compared with traditional WMNs mainly based on our routing algorithm.

\subsection{Simulation settings}

In the simulation, the wireless sub network of Optical-wireless and the traditional WMN use the same network topology for all simulated instances for the sake of fair comparison. The difference between Optical-wireless networks and traditional WMNs resides in the connections between gateways. In our simulation, there are 73 wireless mesh routers which are randomly placed in the $300 \times 300 \mathrm{~m}^{2}$ square region. Each router is equipped with a single radio that works on the single channel as in the IEEE 802.11a. The channel capacity is $c=54 M b p s$. Every node has a transmission range $R_{t}=30 \mathrm{~m}$ and the ratio $\beta$ between $R_{i}$ and $R_{t}$ is set to 1 . In general, we uniformly deploy 5 gateways (ONUs) in the square region and assume that any two gateways are beyond the transmission range of each other.

\subsection{Simulation results}

We compare the network throughputs obtained by both wireless mesh and optical-wireless networks at different loads of network. We take the number of users accessing the network is the load of the network. Suppose if there are 10 source, destination pairs are participating in communication we route 10 links as per the routing algorithm, then we calculate the number of interference links to each link and we calculate the resulting throughput that can be achieved by each link. The sum of individual throughputs is the total network throughput at that percentage of load. At the same load we may get different throughput value because the interference experienced by each link is not only depends on load of the network but also on how the communication links are distributed in the network. If the links are distributed in dense spacing we get lesser throughput than if the links are distributed with wide spacing at the same load.

We calculate the network throughput in the same manner for both wireless mesh and opticalwireless networks, but the difference is at how the traffic is routed in a link. Figure.4. compares the obtained network throughputs for both wireless mesh and optical-wireless networks. We can see that network throughput obtained in optical-wireless network is always much better than wireless mesh network. 
International Journal of Distributed and Parallel Systems (IJDPS) Vol.3, No.3, May 2012

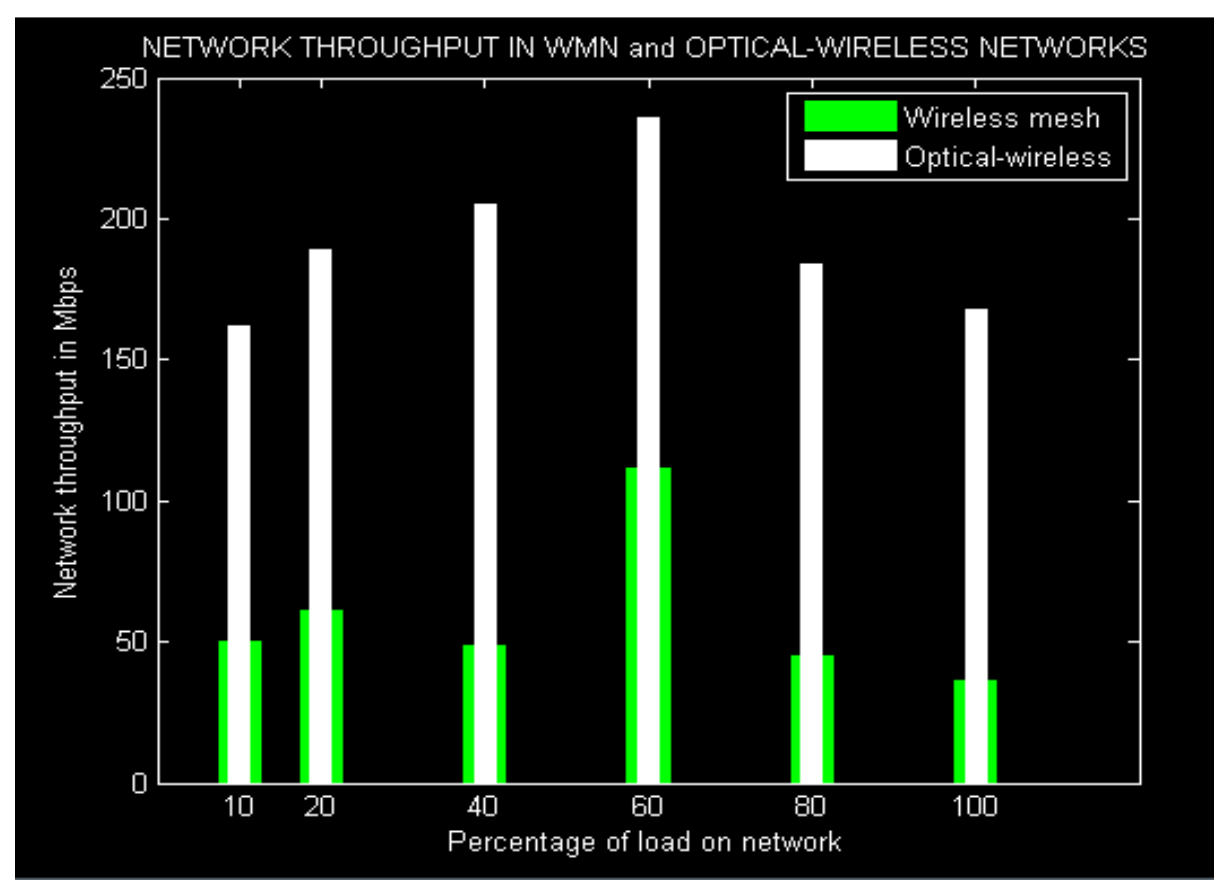

Figure 4. Network throughput in WMN and optical-wireless networks

\section{Conclusion}

This paper proposes a method to improve the network throughput in wireless mesh networks. As the wireless hops increases, interference in the network will increase. By integrating the PON with wireless mesh network we can reduce the number of wireless hops, resulting in decreased interference and increased network throughput.

\section{REFERENCES}

[1] I. Akyildiz, "A survey on wireless mesh networks", IEEE Radio Commun. Vol. 43, No. 9, Sep. 2005, pp.S23-S30.

[2] Chang-Hee Lee, Wayne V. Sorin, "Fiber to the Home Using a PON infrastructure", Journal of light wave technology, Vol. 24, No. 12, Dec. 2006, pp. 4568-4583.

[3] S. Sakar et al., "Hybrid Wireless-Optical Broadband-Access Network(WOBAN): A Review of Relevant Challenges", Journal of Light wave Technology, Vol.25, No.11, Nov. 2007, pp. 3329-3340.

[4] S. Sarkar et al, "A Mixed Integer Programming Model for Optimum Placement of Base Stations and Optical Network Units in a Hybrid Wireless-Optical Broadband Access Network (WOBAN)", IEEE WCNC 2007, pp. 3907-3911.

[5] S. Sarkar et al., 'Towards global optimization of multiple ONUs placement in hybrid optical-wireless broadband access networks", in Proc. COIN, Jul. 2006, pp.65-67.

[6] Saurabh Bhandari, "Hybrid Optical Wireless Networks", ICNICONSMCL 2006.

[7] S. Sarkar, H. Yen, S. Dixit, and B. Mukherjee, 'DARA: Delay aware routing algorithm in a hybrid wireless-optical broadband access network (WOBAN)", in Proc. IEEE ICC, Jun. 2007, pp.2480-2484. 
International Journal of Distributed and Parallel Systems (IJDPS) Vol.3, No.3, May 2012

[8] Sayeem Reaz et al., "CaDAR: an Efficient Routing Algorithm for Wireless-Optical Broadband Access Network", in Proc. ICC 2008, pp.5191-5195.

[9] P. Gupta, P. R. Kumar, 'The capacity of wireless networks", IEEE Transactions on Information Theory, Vol. 46(2000), pp. 388-404.

[10] Jian Tang et al., "Maximum Throughput and Fair Bandwidth Allocation in Multi-Channel Wireless Mesh Networks", IEEE 2006.

[11] M. Alicherry, R. Bhatia, L. Li, "Joint Channel Assignment and Routing for Throughput Optimization in Multiradio Wireless Mesh Networks", ACM MobiCom 2005, pp.58-72.

[12] M. Kodialam and T. Nandagopal, "Characterizing the Capacity Region in Multi-Radio Multi-Channel Wireless Mesh Networks", ACM MobiCom 2005, pp.73-87.

[13] Wei-Tao Shaw, "Hybrid Architecture and Integrated Routing in a Scalable Optical Wireless Access Network", JOURNAL OF LIGHTWAVE TECHNOLOGY, Vol. 25, No. 11, Nov. 2007, pp.3443-3451.

\section{Authors}

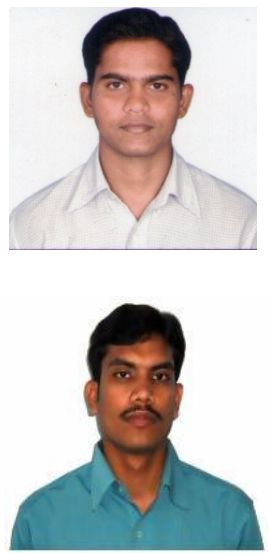

Chakrapani gadde received his B.tech from Lakireddy Balireddy College of engineering in 2008. Currently he is pursuing his M.tech from SIR C R Reddy College of engineering college, Eluru west Godavari district, Andhrapradesh. His areas of interest are wireless communications and computer networking. He has published two papers in international journals relating to "Mitigation of near far effect in GPS receivers" and "Analysis of Energy detection Algorithm in cognitive radio systems".

Chandrasekhar Korumilli received his B.tech from Pragati Engineering College in 2008. Currently he is pursuing his M.tech from SIR C R Reddy College of engineering college, Eluru west Godavari district, Andhrapradesh. His areas of interest are wireless communications, computer networking and Cognitive Radio. He has published a paper in international journals relating to "Analysis of Energy detection Algorithm in cognitive radio systems".

I.Hemalatha received B.tech from Sir CRR College of Engineering, M.tech from JNTU Kakinada. Currently she is Associate Prof at Sir C R Reddy College of Engineering, Eluru west Godavari district, Andhrapradesh. She is having Twelve years of teaching experience and guided many students during this period. Miss.Hemaleta has published many papers and took part in many conferences. 